\title{
COMPARISON OF TWO PARENT-COMPLETED QUESTIONNAIRES FOR THE IDENTIFICATION OF CHILDREN AT RISK FOR AUTISM SPECTRUM DISORDER IN THE PRETERM POPULATION
}

H. Wong ${ }^{1}$, A. Huertas-Ceballos ${ }^{2}$, F.M. Cowan ${ }^{3}$, N. Modi ${ }^{1}$, Medicines for Neonates Investigator Group

${ }^{1}$ Section of Neonatal Medicine, Imperial College London, ${ }^{2}$ Neonatal Unit, University College London

Hospital NHS Foundation Trust, ${ }^{3}$ Departments of Paediatrics and Imaging Sciences, Imperial College London, London, UK

Background: The Bayley Scales of Development Social-Emotional questionnaire (BayleySE,PsychCorp,2006) and the Quantitative Checklist for Autism in Toddlers (Q-CHAT,Allison et al,2008) are newly-developed parent-completed questionnaires providing quantitative measures of early childhood socialcommunication difficulty. They aim to identify children "at-risk" for autism spectrum disorder (ASD).

Aim: To compare agreement between Bayley-SE and Q-CHAT in preterm children.

Methods: The parents of children born at $\leq 29^{+6}$ weeks gestation in a study evaluating routinely collected neurodevelopmental data were requested to complete the Bayley-SE and Q-CHAT. Scores were compared with population norms using Student's t-test. Children with scores $>2$ SD from the mean were considered "at risk" for ASD. Agreement between the questionnaires in identifying these children was measured using Cohen's kappa statistic.

Results: Questionnaires have been completed by the parents of 53 children (mean [SD] gestation 26.5 [1.6] weeks, mean corrected age at assessment 24.1 [2.6] months). Scores were lower in the Bayley-SE (mean composite score 93.2 [19.7]) and higher in the Q-CHAT (mean 36.8 [7.9]) compared to reported population means $(p<0.01)$, indicating greater autistic traits. Q-CHAT classified $12(22.6 \%)$ and Bayley-SE classified 5 $(9.4 \%)$ children as "at-risk" for ASD; only 3 children were identified by both questionnaires (kappa $=0.25$ [95\%CI 0-0.55]).

Conclusions: Parents of preterm children report greater early childhood social-communication difficulties than in the general population. There was poor agreement between Bayley-SE and Q-CHAT in classifying children "at risk" for ASD. Reasons may be the different emphasis placed on reciprocal social interaction in the Bayley-SE and on communication and stereotypical behaviour in the Q-CHAT. 